\title{
Inhibition of c-FLIP expression by miR-512-3p contributes to Taxol-induced apoptosis in hepatocellular carcinoma cells
}

\author{
FENG CHEN $^{1}$, HAI-HONG ZHU ${ }^{1}$, LIN-FU ZHOU $^{2}$, SHAN-SHAN WU $^{1}$, JING WANG $^{1}$ and ZHI CHEN ${ }^{1}$ \\ ${ }^{1}$ State Key Laboratory of Infectious Disease Diagnosis and Treatment, First Affiliated Hospital, College of Medicine, \\ Zhejiang University; ${ }^{2}$ Department of Cell Biology, College of Medicine, Zhejiang University, Hangzhou 310003, P.R. China
}

Received December 11, 2009; Accepted February 8, 2010

DOI: $10.3892 /$ or_00000784

\begin{abstract}
Dysregulation of the antiapoptotic protein cellular FLICE-like inhibitory protein (c-FLIP) has been proven to be associated with tumorigenesis and progress of most human cancers. However, its aberrant expression is poorly elucidated. MicroRNAs (miRNAs) are small non-coding RNAs that are involved in tumorigenesis through negatively regulating gene expression. Our study disclosed that c-FLIP was overexpressed in HepG2 hepatocellular carcinoma cells and downregulation of c-FLIP enhanced Taxol-induced apoptosis. Taxol induction significantly decreased the protein level of c-FLIP. While no decrease in c-FLIP mRNA level was observed, indicating Taxol decreased c-FLIP expression through a posttranscriptional mechanism. miR-512-3p was a predicted suppressor of c-FLIP and exhibited an opposite expression manner to c-FLIP before and after Taxol induction. Luciferase report assay demonstrated miR-512-3p negatively regulated c-FLIP expression via a conserved miRNA-binding site in 3' untranslated region (3'UTR) of c-FLIP. The decrease of c-FLIP protein due to transfection of miR-512-3p further validated the inhibitory effect of miR-512-3p on c-FLIP. Additional transfection of miR-512-3p remarkably promoted Taxol-induced apoptosis, confirming its involvement in apoptosis. In summary, our study disclosed a novel regulatory mechanism that down-regulation of c-FLIP by miR-512-3p contributed to Taxol-induced apoptosis. Importantly, the pivotal role of miR-512-3p in determining c-FLIP abundance helps to broaden the implications for cancer therapy by developing small molecules to directly target c-FLIP at mRNA level.
\end{abstract}

Correspondence to: Dr Zhi Chen, State Key Laboratory of Infectious Disease Diagnosis and Treatment, First Affiliated Hospital, College of Medicine, Zhejiang University, Hangzhou 310003 , P.R. China

E-mail: zju.zhichen@gmail.com

Key words: cellular FLICE-like inhibitory protein, miR-512-3p, Taxol, apoptosis

\section{Introduction}

Protection from death receptor (DR)-mediated apoptosis has been proposed as an important step in the development of malignancy. An important regulator of DR-induced death is the cellular FLICE-like inhibitory protein (c-FLIP). c-FLIP has homology to caspase- 8 and -10 but lacks their protease activity and thereby prevents the activation of procaspase- 8 and potently inhibits apoptosis mediated by death receptors $(1,2)$. As an important antiapoptotic protein, c-FLIP has been shown to be overexpressed in human tumor cells, including colorectal carcinoma, hepatocellular carcinoma, pancreatic carcinoma, and prostate carcinoma (3-7). Dysregulation of c-FLIP expression has been proven to be one of the major determinants of the resistance to death ligands such as FasL and TRAIL, which suggests that targeting c-FLIP is an appealing way to anticancer therapy (8-10). Cisplatin, doxorubicin, actinomycin D, camptothecin, and Trichostatin A have been known to lower c-FLIP expression at transcriptional level and to sensitize the resistant tumor cells to death receptor-mediated apoptosis (11-15). However, due to limited information about c-FLIP regulation, the agents directly targeting c-FLIP at mRNA or protein levels have not yet been developed.

Taxol, as a DNA-damaging agent, just like cisplatin and doxorubicin, is one of the most active cancer chemotherapeutic agents and is effective against several human tumors including ovarian, breast, non-small cell lung tumors, and head and neck carcinomas (16-18). It is evident that Taxolininduced apoptosis is primarily through caspase- 8 and -10 activation, which indicated that Taxol could prevent c-FLIP expression to facilitate caspase- 8 activation and apoptosis signaling $(19,20)$. However, the regulation of Taxol on c-FLIP expression and its molecular mechanisms are still poorly understood.

In our study, we demonstrated that during the process of Taxol-induced apoptosis, the decrease of c-FLIP expression by Taxol was through a post-transcriptional mechanism. We verified that it was miR-512-3p that inhibited c-FLIP expression via directly binding to c-FLIP 3'UTR. The findings implied that inhibition of c-FLIP by miR-512-3p might contribute to Taxol-induced apoptosis in hepatocellular carcinoma cells. Our study suggested that, compared to those agents that prevented the transcription of c-FLIP by blocking 
the corresponding signaling pathways, developing small molecules that directly targeting c-FLIP at mRNA level is expected to be efficient and valuable for cancer therapy.

\section{Materials and methods}

Cell culture and reagents. HepG2 cells were cultured at $37^{\circ} \mathrm{C}$ with $5 \% \mathrm{CO}_{2}$ in Dulbecco's modified Eagle's medium supplemented with $10 \% \mathrm{FBS}, 100 \mathrm{U} / \mathrm{ml}$ penicillin and $100 \mu \mathrm{g} /$ $\mathrm{ml}$ streptomycin. Taxol (paclitaxel, Sigma Co., St. Louis, MO) was dissolved in dimethyl sulfoxide (DMSO) at a concentration of $0.1 \mu \mathrm{M}$ as a stock.

Vectors, Stealth ${ }^{T M}$ siRNAs and microRNA mimics. pGL3FLIP-3'UTR reporter plasmid (designated as pGL3-F) was constructed as follows: the 3'UTR sequence of FLIP was amplified by RT-PCR from HepG2 cDNA using specific primers (forward: 5'-gaaaccaaaaggctgggcgt-3'; and reverse: 5'-cccaggttggtaagc-3'). This fragment was cloned into pGL3 vector downstream of the luciferase gene. We also constructed pGL3-FLIP-3'UTR mutant, in which two sites of the seed sequence was deleted and referred to as pGL3-FM. miR-512$3 \mathrm{p}$ and negative control mimics were synthesized from Dharmacon and dissolved in DEPC-treated $\mathrm{H}_{2} \mathrm{O}$ at a concentration of $20 \mathrm{pmol} / \mu \mathrm{l}$ as a stock. Stealth siRNAs targeting FLIP were synthesized from Invitrogen and dissolved in DEPC-treated $\mathrm{H}_{2} \mathrm{O}$ at a concentration of $20 \mathrm{pmol} / \mu 1$ as a stock.

Semi-quantitative RT-PCR. Total RNA was extracted from HepG2 cells using the TRIzol reagent (Invitrogen, CA, USA). The primers for amplification were: c-FLIP, forward 5'-ggga gaagtaaagaacaaag-3' and reverse 5'-cgtaggcacaatcacagcat-3'; ß-actin, forward 5'-gaaaatctggcaccacacct-3' and reverse 5'-ggc cggactcgtcatactc-3'. PCR products were electrophoresed on $1 \%$ agarose gels containing ethidium bromide and visualized under UV transilluminator.

Real-time PCR for miRNA. Total RNA was extracted from HepG2 cells using the TRIzol reagent (Invitrogen). Real-time PCR was performed with the TaqMans microRNA assays (Applied Biosystems) in the ABI PRISM ${ }^{\circledR} 7500$ Real-time PCR system (Applied Biosystems). The mean Ct was determined from triplicate PCRs. miR-512-3p expression was calculated relative to $18 \mathrm{~S}$ rRNA. The primer sequences were: miR-512-3p, forward 5'-uugcacuggcucagucuggc-3' and reverse 5'-cctcagtcattggacctcag-3'; 18S, forward 5'-ctacca catccaaggaagcca-3' and reverse 5'-ttttcgtcactacctccccg-3'.

Western blotting. Total protein from HepG2 cells was extracted using RIPA buffer $(1 \mathrm{mM} \mathrm{MgCl} 2,10 \mathrm{mM}$ Tris- $\mathrm{HCl}$ pH 7.4, 1\% Triton X-100, 0.1\% SDS, 1\% NP-40) and measured for total protein concentration using Bradford reagent (Bio-Rad, Hercules, CA). Protein extracts $(20 \mu \mathrm{g})$ were separated on $10 \%$ SDS-PAGE gels and transferred to nitrocellulose membranes. Before staining, unspecific sites were blocked in 5\% non-fat milk at room temperature for $1 \mathrm{~h}$. The rabbit polyclonal c-FLIP antibody (1:1000; Santa Cruz Biotechnology) was used to evaluate c-FLIP expression. The secondary horseradish peroxidase-conjugated antibody was detected using ECL plus Western blotting detection reagents

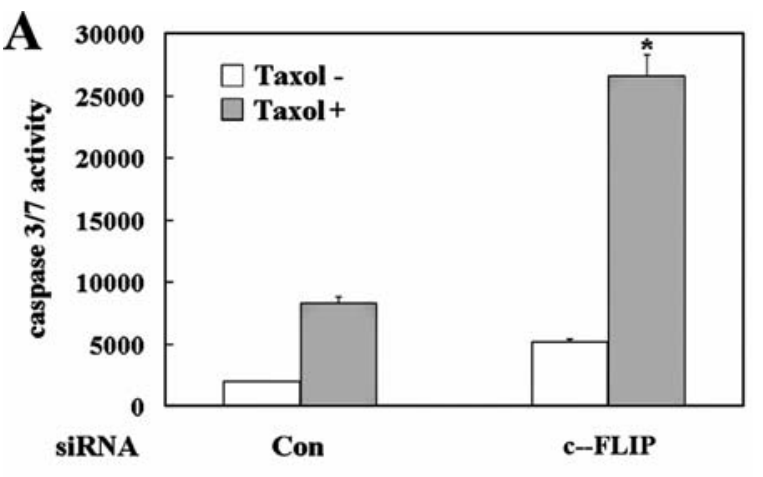

B

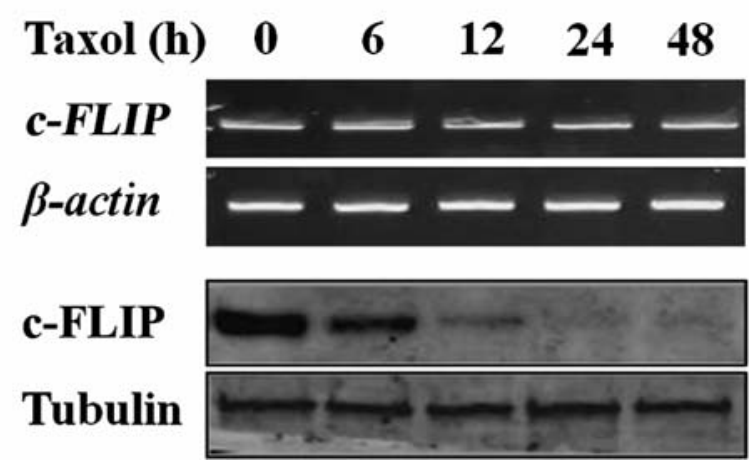

Figure 1. c-FLIP participated in Taxol-induced apoptosis. (A) HepG2 cells were seeded in 96-well plates and transfected with siRNAs specifically targeting FLIP, then treated with Taxol (100 nM) for 24 h. Caspase-3 activities were measured using a Caspase-Glo 3/7 assay kit. Error bars represent $\mathrm{SD}$ and are obtained from three independent experiments; ${ }^{\mathrm{p}}<0.01$. (B) HepG2 cells were plated in 6-well and incubated with Taxol. At the indicated time points $(0,6,12,24$, and $48 \mathrm{~h})$, total RNA and proteins were extracted. RT-PCR (upper panel) and Western blot (lower panel) were performed to detect the expression of c-FLIP. B-actin and Tubulin were used as internal controls. Results presented here are representative of three different experiments.

(Amersham Biosciences). As a loading control, the B-tubulin expression level was measured using rabbit polyclonal antiß-tubulin antibody (Santa Cruz Biotechnology).

Luciferase gene assay. The HepG2 cells were cultured in 24well plates for $24 \mathrm{~h}$, then transfected with $400 \mathrm{ng}$ of pGL3-F (or pGL3-FM), and different dose of miR-512-3p mimics 20, 40,80 pmol) using DharmaFECT ${ }^{\circledR}$ Duo Transfection Reagent (Dharmacon). pBIND plasmids (50 ng) were cotransfected to normalize for transfection efficiency. At $48 \mathrm{~h}$ post-transfection, the firefly and Renilla luciferase activities were measured using the dual-luciferase reagent assay kit (Promega, Madison, WI) according to the manufacturer's instructions. Three independent experiments were performed in triplicate.

Cell apoptosis assay. HepG2 cells were plated in 6-well plates, then transfected with siRNAs targeting c-FLIP for $24 \mathrm{~h}$ and treated with Taxol. After $24 \mathrm{~h}$, cells were incubated with propidium iodide exclusion and Annexin V-fluorescein isothiocyanate (FITC), and analyzed by flow cytometry.

Caspase activity assay. HepG2 cells were plated in replicates of 6 in 96-well plates at a density of $5 \times 10^{3}$ cells/well for $24 \mathrm{~h}$, 

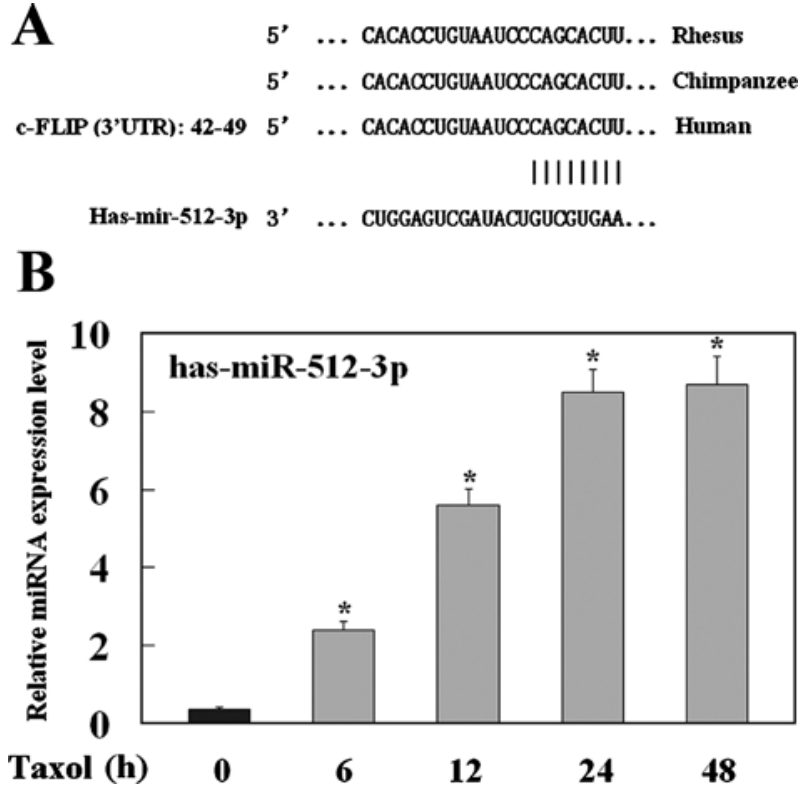

Figure 2. miR-512-3p as a putative inhibitor of c-FLIP by bioinformatics analyses. (A) A schematic representation illustrating the c-FLIP mRNA 3'UTR putative sites targeted by miR-512-3p predicted by Pictar and TargetScanS database. (B) HepG2 cells were plated in 6-well plates and treated with $100 \mathrm{nM}$ of Taxol. At the indicated time points $(0,6,12,24$, $48 \mathrm{~h}$ ), total RNAs were extracted and real-time PCR was performed to examine the miR-512-3p level. Samples from three independent experiments are analyzed and the data are expressed as means $\pm \mathrm{SD} ;{ }^{*} \mathrm{p}<0.01$

then transfected with siRNAs targeting c-FLIP for $24 \mathrm{~h}$ and treated with Taxol. Annexin V-FITC positive cells were determined by flow cytometry. For apoptotic effects of miR$512-3 p$, cells were transfected with miR-512-3p and control mimics for $24 \mathrm{~h}$ and subsequently treated with Taxol. After indicated treatment, $50 \mu 1$ of Caspase-Glo 3/7 Reagent (Promega) were added into each well and incubated for $1 \mathrm{~h}$. The luminescence of each well was measured in the GENios Pro Multifunctional Reader from Tecan.

\section{Results}

Knockdown of c-FLIP enhanced Taxol-induced apoptosis. It has been recently reported that Taxol-induced apoptosis is FADD dependent, combined with the inhibitory role of c-FLIP in death receptors-induced apoptosis, we wonder whether knockdown of c-FLIP sensitized hepatocellular carcinoma cells to Taxol induction. As shown in Fig. 1A, Taxol induction caused a significant increase of capase-3/7 activity by 3.3 -fold in siFLIP cells, compared to its effects in siCon cells, which demonstrated that c-FLIP was involved in Taxol-induced apoptosis.

Next, we tested the expression of c-FLIP in HepG2 cells following Taxol treatment. As shown in Fig. 1B, the protein level of c-FLIP was down-regulated dramatically by Taxol in a time-dependent manner, especially at $12 \mathrm{~h}$ of Taxol incubation; while, its mRNA level showed slight alteration, indicating that Taxol controls c-FLIP expression through a post-transcriptional mechanism.

miR-512-3p is a potent suppressor of c-FLIP predicted by bioinformatics analyses. MicroRNAs regulate a variety of

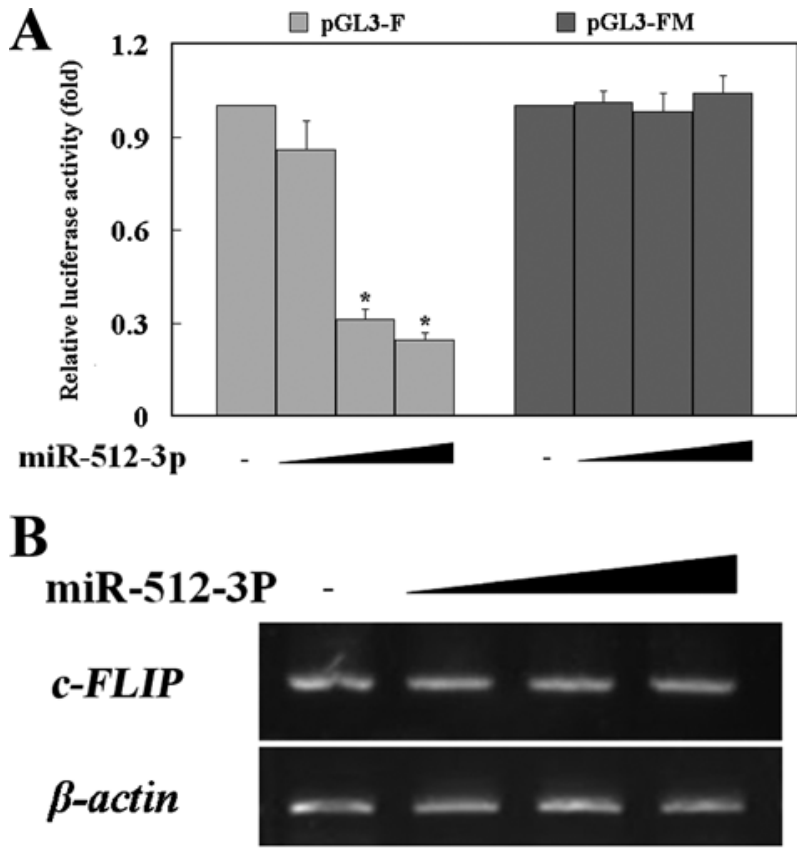

Figure 3. Negative regulation of miR-21 on c-FLIP expression. (A) HepG2 cells were plated in 24-well plates, and cotransfected with pGL3-FLIP3'UTR (wild-type or mutant, designated as pGL3-F and pGL3-FM) and different dose of miR-512-3p mimic (20, 40, and 80 pmol). Luciferase activity was determined $48 \mathrm{~h}$ after transfection. Activity of the firefly luciferase was normalized to that of the renilla luciferase and expressed as relative luciferase unit (RLU). Error bars represented SD and were obtained from three independent experiments. Student's t-test was applied for analysis of significant difference; ${ }^{*} \mathrm{p}<0.05$. (B) HepG2 cells were plated in 6-well plates and transduced with different dose of miR-512-3p mimics $(20,40$, and $80 \mathrm{pmol}$ ) and control mimic. At 48 -h post-transfection, the c-FLIP RNA and protein levels were assessed by RT-PCR and Western blotting.

cellular activities through regulation of the expression of multiple target genes via repressing translation or promoting mRNA decay (21-23). To examine the involvement of microRNAs in c-FLIP expression during the process of Taxol incubation, we focused on the identification of putative microRNAs by Pictar and TargetScan software. There were 15 miRNAs that were predicted to bind to the 3'UTR of human c-FLIP by the two softwares. Based on published studies, majority of predicted targets function as anti-apoptotic proteins, for example miR-150, miR-17-5p, miR-93 and miR221. Among them, miR-512-3p is the unique candidate because it has been verified as a pro-apoptotic tumor suppressor in variety of carcinomas (24). Fig. 2A shows that the conserved target sites for miR-512-3p in the 3'UTR of cFLIP is at nucleotides 1937-1942, conservation of which was also observed in other species. To characterize miR-512-3p was a putative suppressor of c-FLIP, we examined its expression in Taxol-treated cells. The expression level of miR-512-3p was nearly undetectable in HepG2 cells. While, its expression elevated rapidly at $6 \mathrm{~h}$ of Taxol incubation and nearly reached the maximum $24 \mathrm{~h}$ later (Fig. 2B), hinting that miR-512-3p expression was triggered by Taxol.

Negative regulation of $m i R-512-3 p$ on $c-F L I P$. We next examined the effect of miR-512-3p on endogenous c-FLIP expression. To test this, we subcloned the c-FLIP 3'UTR (designated as pGL3-F) downstream of luciferase gene. 

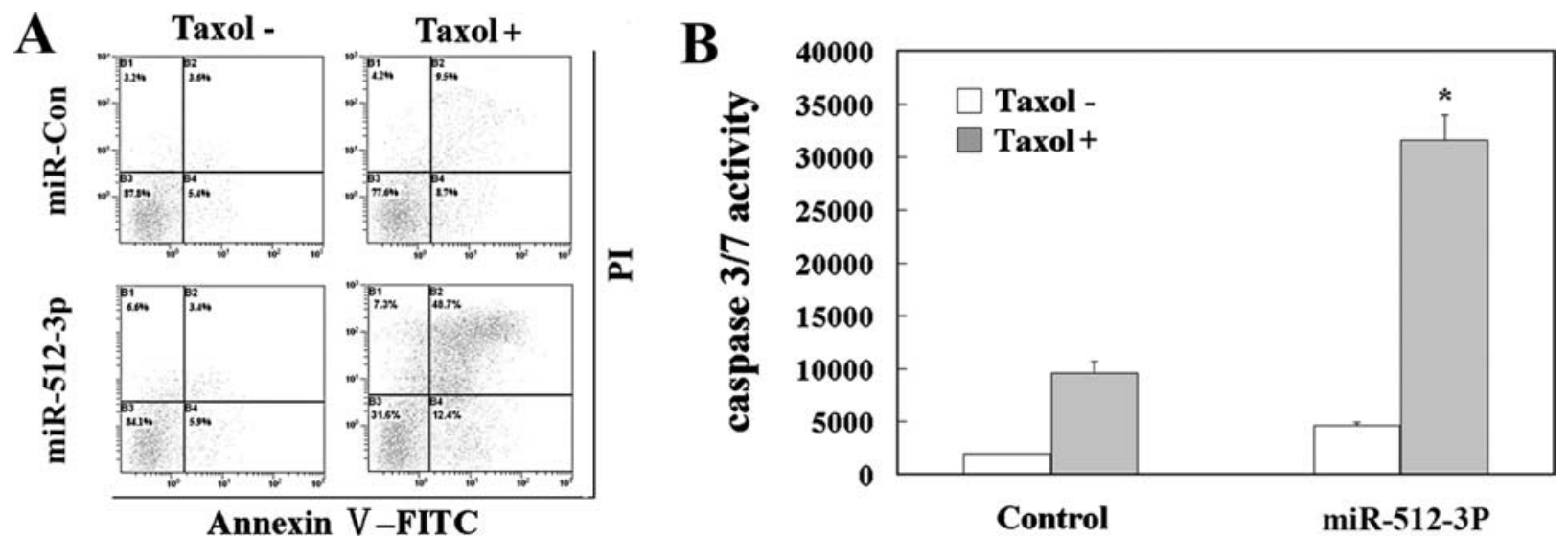

Figure 4. miR-512-3p enhances Taxol-induced apoptosis. (A) HepG2 cells were plated in 6-well plates and transfected with miR-512-3p and control mimics for $24 \mathrm{~h}$ and subsequently treated with Taxol. After $24 \mathrm{~h}$ of incubation, the apoptotic cells were measured by PI and Annexin V-FITC staining and analyzed by flow cytometry. Percentages in the figures represent the mean from three independent experiments. (B) HepG2 cells were plated in 96-well plates and treated as described above. Caspase-3 activities were measured using a Caspase-Glo 3/7 assay kit (Promega). Error bars represent SD and were obtained from three independent experiments; ${ }^{*} \mathrm{p}<0.01$.

Cotransfection of cells with pGL3-F plus the miR-512-3p decreased the luciferase activity in a dose-dependent manner. To further determine the suppression of miR-512-3p on c-FLIP by binding to c-FLIP 3'UTR, the seed region of c-FLIP 3'UTR was deleted and subcloned downstream of luciferase gene (designated as pGL3-FM). As we expected, the luciferase activity in pGL3-FM was unchanged by overexpression of miR-512-3p (Fig. 3A). Our data demonstrated that negative post-transcriptional regulation of miR-512-3p on c-FLIP was dependent on miR-512-3p target sites in c-FLIP 3'UTR. To check if miR-512-3p actually affected c-FLIP expression in cellular environment, we analyzed the consequences of the ectopic expression of miR-512-3p. We found that the protein level of c-FLIP was increased in a dose-dependent manner in the miR-512-3p-transduced cells, while no significant difference in c-FLIP mRNA level was observed between miR-512-3p-transduced cells and control cells (Fig. 3B). These data suggested that miR-512-3p specifically downregulated c-FLIP expression at the post-transcriptional level.

miR-512-3p restoration promotes Taxol-induced apoptosis. Considering the negative regulation of miR-512-3p on c-FLIP expression, combined with its increased expression after Taxol induction, we next evaluated the role of miR-512-3p in the process of Taxol-induced apoptosis. In the presence of Taxol, $61.1 \%$ of cells transfected with miR-512-3p mimics were Annexin V positive, compared with $18.2 \%$ Annexin V positivity in Taxol-treated negative control cells (Fig. 4A, right panels).

MiR-512-3p overexpression in combination with $24 \mathrm{~h}$ of Taxol treatment resulted in increased activation of caspase- 3 compared to control cells (Fig. 4B, p<0.01). These findings suggested that miR-512-3p may function as a pro-apoptotic activator of cell death.

\section{Discussion}

c-FLIP is a recently identified intracellular inhibitor of caspase- 8 activation, which inhibits the apoptosis signaling mediated by the death receptors $(2,25,26)$. Since c-FLIP is recognized more recently and the detailed molecular mechanisms on its regulation are deficient, thus developing small molecular inhibitors that aim at c-FLIP seems to be left behind in comparison to antiapoptotic proteins $\mathrm{Bcl}-2$, IAP and MDM2, functions and molecular mechanisms of which are well-documented (27-29). Considering the characterized modulators regulate the expression of these antiapoptotic proteins at transcriptional, post-transctiptional, and translational levels, many small molecule inhibitors are designed to target Bcl-2, IAP, and MDM2 at either mRNA or protein levels are now in preclinical and early clinical trials (27-29). Cisplatin, doxorubicin, actinomycin D, and camptothecin have been proven to sensitize tumor cells to death receptor-mediated apoptosis by inhibiting the transcription of c-FLIP (11-15). However, small molecules, such as antisense oligonucleotides or miRNAs, which could directly aim at the mRNA of the targets, are seldom reported due to lack of valuable data on post-transcriptional regulation of c-FLIP.

Interestingly, our study observed that in hepatocellular carcinoma cells, Taxol induction significantly decreased the protein level of c-FLIP. While no decrease of c-FLIP mRNA level were observed, which indicated that Taxol decreased c-FLIP expression via a post-transcriptional mechanism. Our data are consistent with several published results. For example, PPAR $\gamma$ ligands sensitized tumors to TRAILinduced apoptosis by reducing c-FLIP through inducing ubiquitination and proteasome-dependent degradation of c-FLIP (30). Poukkula et al reported that c-FLIP could be down-regulated by a transcription-independent mechanism involving protein ubiquitylation (31). It has been reported that Taxol triggered caspase-8- and caspase-10-dependent apoptosis in human lymphoblastic leukemia cell line by inhibiting protein level of c-FLIP rather than mRNA level (32). Our data, combined with others, suggested that posttranscriptional regulation may be a common mechanism of c-FLIP expression.

MicroRNAs are a group of non-coding, single-stranded RNAs that negatively modulate gene expression at the 
post-transcriptional level through association with the 3'UTR of protein coding genes and subsequent induction of translation inhibition or transcript destabilization $(22,23)$. Accumulating evidence demonstrate that microRNAs contribute to cancer development by acting on oncogenes or tumor suppressor genes (33-36). For example, BCL2 are frequently highly expressed in chronic lymphocytic leukemia. Targeting BCL2 by miR-15a and miR-16 significantly enhance chemotherapyinduced apoptosis (37). Compared to oncogenes, p27 acts as a tumor suppressor and its expression is often disrupted in human cancers. High activity of miR-221 and miR-222 is required to maintain oncogenic state in cancer cells by inhibiting p27 expression (38).

Considering the inverse correlation between miR-512-3p and c-FLIP by Taxol induction and miRNAs as potent regulators of gene expression, we hypothesized that downregulation of c-FLIP might be mediated by microRNAs. By using PicTar and TargetScan databases, we identified 15 putative targets. Among them, miR-512-3p was the only one that was able to induce apoptosis, which is contrary to cFLIP function. Further data validated the association between miR-512-3p and the 3'UTR of c-FLIP mRNA and also demonstrated that the seed sequence strongly contributed to the miRNA-mRNA interaction which mediated the posttranscriptional inhibition on c-FLIP expression. Furthermore, we found that miR-512-3p over-expression inhibited c-FLIP mRNA level rather that protein level, which indicated that miR-512-3p controlled c-FLIP translation, but not mRNA stability.

Saito et al recently reported that miR-512-3p was silenced in gastric cancer cells (24). Epigenetic activation of silenced miR-512-3p resulted in apoptosis of gastric cancer cells by inhibiting the oncogene Mcl-1, which for the first time indicated that miR-512-3p could promote apoptosis. In agreement with this, our study verified that overexpression of miR-512-3p enhanced Taxol-induced apoptosis by inhibiting c-FLIP expression, which suggests that miR-512-3p may function as a novel tumor suppressor. Moreover, the inverse correlation between miR-512-3p and c-FLIP suggests that lower expression of miR-512-3p resulting in dysregulation of c-FLIP expression may lead to tumorigenesis of hepatocellular carcinoma.

In conclusion, our study disclosed that microRNA-512 functioned as a proapoptotic protein and inhibition of c-FLIP by miR-512-3p contributed to Taxol-induced apoptosis in hepatocellular carcinoma cells. The present study not only helps us to understand the molecular mechanism of tumorigenesis, but also provides a potent strategy for the treatment of carcinomas by developing small molecule inhibitors directly targeting c-FLIP at the levels of mRNA and protein.

\section{Acknowledgements}

The work was supported by The National Basic Research Program (973 Program, 2007CB512905) and The State S\&T Projects (2008ZX10002-007).

\section{References}

1. Debatin KM and Krammer PH: Death receptors in chemotherapy and cancer. Oncogene 23: 2950-2966, 2004.
2. Thorburn A: Death receptor-induced cell killing. Cell Signal 16: 139-144, 2004.

3. Hernandez A, Wang QD, Schwartz SA and Evers BM: Sensitization of human colon cancer cells to TRAIL-mediated apoptosis. J Gastrointest Surg 5: 56-65, 2001.

4. Elnemr A, Ohta T, Yachie A, et al: Human pancreatic cancer cells disable function of Fas receptors at several levels in Fas signal transduction pathway. Int J Oncol 18: 311-316, 2001.

5. Okano H, Shiraki K, Inoue H, Kawakita T, Yamanaka T, Deguchi M, Sugimoto K, Sakai T, Ohmori S, Fujikawa K, Murata K and Nakano T: Cellular FLICE/caspase-8-inhibitory protein as a principal regulator of cell death and survival in human hepatocellular carcinoma. Lab Invest 83: 1033-1043, 2003.

6. Zhang X, Jin TG, Yang H, DeWolf WC, Khosravi-Far R and Olumi AF: Persistent c-FLIP(L) expression is necessary and sufficient to maintain resistance to tumor necrosis factor-related apoptosis-inducing ligand-mediated apoptosis in prostate cancer. Cancer Res 64: 7086-7091, 2004.

7. Mori T, Doi R, Toyoda E, et al: Regulation of the resistance to TRAIL-induced apoptosis as a new strategy for pancreatic cancer. Surgery 138: 71-77, 2005.

8. Djerbi M, Screpanti V, Catrina AI, Bogen B, Biberfeld P and Grandien A: The inhibitor of death receptor signaling, FLICEinhibitory protein defines a new class of tumor progression factors. J Exp Med 190: 1025-1032, 1999.

9. Micheau O, Thome M, Schneider P, et al: The long form of FLIP is an activator of caspase- 8 at the Fas death-inducing signaling complex. J Biol Chem 277: 45162-45171, 2002.

10. Abedini MR, Qiu Q, Yan X and Tsang BK: Possible role of FLICE-like inhibitory protein (FLIP) in chemoresistant ovarian cancer cells in vitro. Oncogene 23: 6997-7004, 2004.

11. Kataoka T. The caspase- 8 modulator c-FLIP. Crit Rev Immunol 25: 31-58, 2005.

12. Longley DB, Wilson TR, McEwan M, et al: c-FLIP inhibits chemotherapy-induced colorectal cancer cell death. Oncogene 25: 838-848, 2006.

13. Yamanaka T, Shiraki K, Sugimoto K, Ito T, Fujikawa K, Ito M, Takase K, Moriyama M, Nakano T and Suzuki A: Chemotherapeutic agents augment TRAIL-induced apoptosis in human hepatocellular carcinoma cell lines. Hepatology 32: 482-490, 2000.

14. Abedini MR, Muller EJ, Brun J, Bergeron R, Gray DA and Tsang BK: Cisplatin induces p53-dependent FLICE-like inhibitory protein ubiquitination in ovarian cancer cells. Cancer Res 68: 4511-4517, 2008.

15. Park SJ, Kim MJ, Kim HB, Sohn HY, Bae JH, Kang CD and Kim SH: Trichostatin A sensitizes human ovarian cancer cells to TRAIL-induced apoptosis by down-regulation of c-FLIPL via inhibition of EGFR pathway. Biochem Pharmacol 77: 1328-1336, 2009.

16. Frederick PJ, Kendrick JE, Straughn JM Jr, Della Manna DL, Oliver PG, Lin HY, Grizzle WE, Stockard CR, Alvarez RD, Zhou T, LoBuglio AF and Buchsbaum DJ: Effect of TRA-8 anti-death receptor 5 antibody in combination with chemotherapy in an ex vivo human ovarian cancer model. Int J Gynecol Cancer 19: 814-819, 2009.

17. Small GW, Shi YY, Higgins LS and Orlowski RZ: Mitogenactivated protein kinase phosphatase-1 is a mediator of breast cancer chemoresistance. Cancer Res 67: 4459-4566, 2007.

18. Sano D, Matsuda H, Ishiguro Y, Nishimura G, Kawakami M and Tsukuda M: Antitumor effects of IDN5109 on head and neck squamous cell carcinoma. Oncol Rep 15: 329-334, 2006.

19. Park SJ, Wu CH, Gordon JD, Zhong X, Emami A and Safa AR: Taxol induces caspase-10-dependent apoptosis. J Biol Chem 279: 51057-51056, 2004.

20. Mielgo A, Torres VA, Clair K, Barbero S and Stupack DG: Paclitaxel promotes a caspase 8-mediated apoptosis through death effector domain association with microtubules. Oncogene 28: 3551-3562, 2009.

21. Ambros V: The functions of animal microRNAs. Nature 431: 350-355, 2004.

22. Cullen BR: Transcription and processing of human microRNA precursors. Mol Cell 16: 861-865, 2004.

23. Lai EC: Micro RNAs are complementary to 3 'UTR sequence motifs that mediate negative post-transcriptional regulation. Nat Genet 30: 363-364, 2002.

24. Saito Y, Suzuki H, Tsugawa H, Nakagawa I, Matsuzaki J, Kanai Y and Hibi T: Chromatin remodeling at Alu repeats by epigenetic treatment activates silenced microRNA-512-5p with downregulation of Mcl-1 in human gastric cancer cells. Oncogene 28: 2738-2744, 2009. 
25. Nam SY, Jung GA, Hur GC, et al: Upregulation of FLIP(S) by Akt, a possible inhibition mechanism of TRAIL-induced apoptosis in human gastric cancers. Cancer Sci 94: 1066-1073, 2003.

26. Krueger A, Baumann S, Krammer PH and Kirchhoff S: FLICE inhibitory proteins: regulators of death receptor-mediated apoptosis. Mol Cell Biol 21: 8247-8254, 2001.

27. Fesik SW: Promoting apoptosis as a strategy for cancer drug discovery. Nat Rev Cancer 5: 876-885, 2005.

28. Reed JC and Pellecchia M: Apoptosis-based therapies for hematologic malignancies. Blood 106: 408-418, 2005.

29. Arkin M: Protein-protein interactions and cancer: small molecules going in for the kill. Curr Opin Chem Biol 9: 317-324, 2005.

30. Kim Y, Suh N, Sporn M and Reed JC: An inducible pathway for degradation of FLIP protein sensitizes tumor cells to TRAILinduced apoptosis. J Biol Chem 277: 22320-22329, 2002.

31. Poukkula M, Kaunisto A, Hietakangas V, et al: Rapid turnover of c-FLIPS is determined by its unique C-terminal tail. J Biol Chem 280: 27345-27355, 2005.

32. Day TW, Najafi F, Wu CH and Safa AR: Cellular FLICE-like inhibitory protein (c-FLIP): a novel target for Taxol-induced apoptosis. Biochem Pharmacol 71: 1551-1561, 2006.
33. Takamizawa J, Konishi H, Yanagisawa K, et al: Reduced expression of the let-7 microRNAs in human lung cancers in association with shortened postoperative survival. Cancer Res 64: 3753-3756, 2004.

34. Calin GA, Sevignani C, Dumitru CD, et al: Human microRNA genes are frequently located at fragile sites and genomic regions involved in cancers. Proc Natl Acad Sci USA 101: 2999-3004, 2004.

35. He L, He X, Lim LP, et al: A microRNA component of the p53 tumour suppressor network. Nature 447: 1130-1134, 2007.

36. Hayashita $\mathrm{Y}$, Osada $\mathrm{H}$, Tatematsu $\mathrm{Y}$, et al: A polycistronic microRNA cluster, miR-17-92, is overexpressed in human lung cancers and enhances cell proliferation. Cancer Res 65: 9628-9632, 2005.

37. Cimmino A, Calin GA, Fabbri M, et al: miR-15 and miR-16 induce apoptosis by targeting BCL2. Proc Natl Acad Sci USA 102: 13944-13949, 2005.

38. Miller TE, Ghoshal K, Ramaswamy B, et al: MicroRNA-221/ 222 confers tamoxifen resistance in breast cancer by targeting p27Kip1. J Biol Chem 283: 29897-29903, 2008. 